\title{
Veri Artırma Tekniklerinin Derin Öğrenmeye Dayalı Yüz Tanıma Sisteminde Etkisi
}

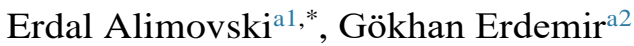 \\ $\mathrm{a}^{1}$ İstanbul Sabahattin Zaim Üniversitesi, Mühendislik ve Doğa Bilimleri Fakültesi, Bilgisayar Mühendisliği Bölümü, İstanbul, Türkiye \\ $\mathrm{a}^{2}$ İstanbul Sabahattin Zaim Üniversitesi, Mühendislik ve Doğa Bilimleri Fakültesi, Elektrik-Elektronik Bölümü, İstanbul, Türkiye
}

İstanbul Sabahattin Zaim Üniversitesi Fen Bilimleri Enstitüsü Dergisi (2021) 3 (1): 76-80

https://doi.org/10.47769/izufbed.880581

(iD) $O R C I D{ }_{1}^{1} 0000-0003-0909-2047 ;{ }^{2} 0000-0003-4095-6333$

\section{YAYIN BILGISII}

Yayın geçmişi:

Gönderilen tarih: 15 Şubat 2021

Kabul tarihi: 17 Mart 2021

Anahtar kelimeler:

Derin Öğrenme

Evrişimsel Sinir Ağları

Yüz Tanıma

Veri Artırma

\section{ÖZET}

Geçtiğimiz son on yılda, Derin Öğrenme, bilhassa Evrişimsel Sinir Ağları (Convolutional Neural Network), Makine Öğrenmesi ve Derin Sinir Ağları'nın en hızlı büyüyen alanıdır. Birçok Derin Sinir Ağı arasında, günümüzde Evrişimsel Sinir Ağları görüntü analizi ve sınıflandırma amaçları için kullanılan ana araçların başında gelmektedir. Evrişimsel Sinir Ağı tabanlı modeller, yüz tanıma görevlerinde yüksek başarı performansı sergilemektedirler. İlgili modellerin başarı performansının yüksek olması oluşturulan mimariye ve tercih edilen hiper-parametrelere bağlıdır. Ek olarak, modellerin eğitildikleri veri setinin boyutu performans üzerinde büyük etkiye sahiptir. $\mathrm{Bu}$ çalışmada ana amacımız, afin dönüşümü (affine transform) yöntemi ile veri artırma işlemini gerçekleştirmek ve bu veri artırma tekniğinin Evrişimsel Sinir Ağlarına dayalı yüz tanıma sistemine etkisini analiz etmektir. Evrişimsel Sinir Ağlarına dayalı yüz tanıma sistemi Destek Vektör Makineleri ve K-En Yakın Komşu sınıflandırma algoritmaları ile uygulanmış, devamına iki algoritmanın performansı karşılaştırılmıştır. Çalışmamızda bütün deneyler Labeled Faces in the Wild (LFW) veri seti üzerinde gerçekleşmiştir. Elde edilen sonuçlar, uygulanan veri artırma tekniğinin, yüz doğrulama işlemi için $\% 1.8$ oranında, yüz sınıflandırma işlemi için \%2.2 (Destek Vektör Makineleri) ve \%2.5 (K-En Yakın Komşu) oranında artış sergilediği gözlemlenmiştir. Gerçekleşen bütün deneyler sonunda, yüz tanıma sistemi doğrulama işleminde $\% 94.4$ oranında doğruluk elde etmiştir. Sınıflandırma işlemlerinde ise sistem, Destek Vektör Makineleri algoritması uygulanarak \%97.1, K-En Yakın Komşu algoritması uygulanarak ise \%96.3 oranında başarı performansı elde etmiştir.

\section{Effects of Data Augmentation Techniques on Face Recognition System Based on Deep Learning}

\begin{tabular}{l}
\hline ARTICLE INFO \\
\hline Article history: \\
Received: 15 February 2021 \\
Accepted: 17 March 2021 \\
\hline Key words: \\
Deep Learning \\
Convolutional Neural Network \\
Face Recognition \\
Data Augmentation
\end{tabular}

* Sorumlu yazar

E-mail adresi:erdal.alimovski@izu.edu.tr (Erdal Alimovski)

\section{ABSTRACT}

In the last decade, Deep Learning particulary Convolutional Neural Networks is the fastest growing area of Machine Learning and Deep Neural Networks. Amongs the many Deep Neural Networks, Convolutional Neural Networks are one of the main tools used for image analysis and classification tasks. Convolutional Neural Network-based models performs high performance in face recognition tasks. The performance of the relevant models depends on their architecture and hyper-parameters. In addition, the size of the dataset in which the models are trained has a large impact on performance. Main goal of this study is to perform data augmentation based affine transform method and to analyze the effect of this data enhancement technique on face recognition system based on Convolutional Neural Networks. Face recognition system based on Convolutional Neural Networks was performed using Support Vector Machines and K-Nearest Neighbor classification algorithms. Following, the performance of the two algorithms was compared. All experiments in our study were carried out on the Labeled Faces in the Wild (LFW) dataset. Obtained results demonstrates that applied data augmentation technique increase the performance of face recognition system, in face verification task for 1.8\%, whereas for classification task 2.2\% for Support Vector Machines and 2.5\% for K-Nearest Neighbor. Finnaly, face recognition system achieved $94.4 \%$ accuracy in verification phase, $97.1 \%$ (Support Vector Machines) and 96.3\% (K-Nearest Neighbor) for classification task. 


\section{Giriş}

Son yıllarda Derin Öğrenme (DÖ) alanı çok hızlı gelişmektedir. Bu gelişmeler yeni stratejilerin ve yapıların ortaya çıkmasına neden olmuştur. Evrişimsel Sinir Ağları (ESA), ilgili alanda en önemli tekniklerin başında gelmektedir. ESA, nesne tanıma, sınıflandırma, görüntü bölme vs. gibi görüntü işleme görevlerinde kullanılan temel araçtır. İlk ESA 1980 yılında Kunihiko Fukushima tarafindan tanitılan "neocognitron" dur (Fukushima, 1988). Ancak, 1998 yılında [LeCun vd., 1998] çalışmasında evrişim çekirdek (kernel) katsayılarını doğrudan elle yazılmış sayıların görüntülerinden öğrenmek için geri yayılımı kullanılmıştır. Böylece öğrenme tamamen otomatik olarak gerçekleşmişti ve manuel katsayı tasarımından daha iyi performans sergilediği gözlemlenmiş. Dolayısıyla, daha geniş görüntü tanıma problemleri ve görüntü türleri yelpazesine uygun olduğu kanıtlanmıştır. Bu yaklaşım, bilgisayar görünüşü'nün (computer vision) temeli olarak literatürde kayıtlara geçti.

Yüz sınıflandırma işlemi oldukça zorlu bir problemdir. Hayatımız boyunca birçok farklı yüz gördüğümüz için, insanoğlu olarak, yüzleri ayırt edici özellikleriyle kolayca tanıyabiliyoruz. Insanoğlun'da var olan bu yetenek, birçok farklı ve zor koşulda bile oldukça sağlam ve etkilidir. İnsan yüzündeki farklı saç modellerini, gözlük, sakal, bıyık gibi birçok farklılı̆̆ı da kolayca algılayabilemkteyiz. Ancak bilgisayarların bu işlemi kolayca algılayabilmesi ve tanıması oldukça zordur. Dolayısıyla, yüz tanıma işlemini gerçekleştirecek bir model oluşturmak çok zor bir sorundur bunun nedeni ise yüzlerin karmaşık, çok boyutlu ve zaman içinde değişime tabi olmalarıdır (Pawar vd., 2017). Literatür'de yüz tanıma sistemlerinin geliştirilmesiyle ilgili birçok araştırma mevcuttur. Genel olarak bu araştırmalar Derin Öğrenme tekniklerine dayalı modern yöntemler ve Geleneksel yöntemler olarak ayırabiliriz. Gelenksel yöntemler genelde geometrik yaklaşımlara dayalıdırlar. Bahse konu yöntemler, yüze ait bazı bölgelerdeki özelliklerden yola çıkarak yüz tanıma işlemini gerçekleştirirler. Modern yöntemler ise derin öğrenme tekniklerine dayanırlar ve yüze ait özellikler model tarafından verilerden seçilir. İlgili yöntemler büyük boyutlu veri setleri ile eğitildiklerinde yüksek başarı performansı sergilemektedirler. Ancak büyük boyutlu veri seti oluşturmak oldukça zor ve zahmetlidir. Dolayısıyla bu sorunu çözmek için literatürde farklı veri artırma teknikleri önerilmiştir. Bir DÖ stratejisi olarak, veri artırma (data augmentation), veri seti örneklerini artırmak için kullanılan ve oldukça olumlu sonuçlar veren bir tekniktir. Veri setlerinin boyutu düşük olduğu ve uygulanan modelin performansı düşük olduğu durumlarda oldukça gerekli bir tekniktir, veri artırma. Ayrıca, aşırı öğrenmeyi (overfitting) azaltır ve veri dağılımını dengelemektedir. Yüz tanıma sistemleri, son yıllarda araştırmacıların ilgi odağı olmuşturlar. Ancak, bilimsel çalışmalara bakıldığında iyi performans sergileyen yüz tanıma sistemi geliştirmek başlangıçta düşünülenden daha zor olduğu görülmektedir. Geometrik veya istatistiksel yaklaşımlara dayalı yüz tanıma sistemleri farklı poz, aydınlatma, gürültü vs. zor koşular altında yüksek performans sergileyememektedirler. İlk yüz tanıma sistemi 1973 yılında geliştirilmiştir (Kanade, 1974). Araştırmacılar, basit görüntü işleme teknikleri uygulayarak yüze ait 16 özellikli bir vektör elde etmiştirler. Devamında, yüz tanıma işlemini gerçekleştirmek için Öklid mesafe hesaplama tekniğini kullanmıştırlar. İlgili çalışmada önerilen yüz tanıma sistemi \%75 doğruluk elde etmiştir. Diğer bir çalışmada, (Kanade T., 1973)'nin çalışmasından esinlenerek araştırmacılar yüze ait özellik sayısını 35'e çıkararak \%95 oranında doğruluk elde etmiştirler (Cox vd., 1996). Geometrik veya istatistiksel bazlı yüz tanıma sistemleri her nekadar kısıtlanmamış ortarmlarda iyi performans sergileseler de kısıtlanmamış ortamlarda doğruluk performansları oldukça düşüktür. Bunun ana nedeni, özelliklerin araştırmacılar tarafindan manuel seçilmesidir. Dolayısıyla bazı seçimler doğru olabilirken, bazıları yanlış olabilmekte ve yüz tanıma sisteminin performansına etki etmektedir. Bu ve buna benzer sorunları çözmek için DÖ dayalı yüz tanıma sistemleri geliştirilmiştir. Günümüzün en popüler ve en yüksek performanslı yüz tanıma sistemi google tarafından geliştirilen FaceNet dir (Schroff vd., 2015). İlgili yüz tanıma sistemi DÖ dayalı geliştirilip 200 milyon görüntü ile eğitilmiş, ve \%99.63 oranında başarı performansı elde etmiştir. FaceNet adlı yüz tanıma sisteminin mimarisi, DeepFace mimarisi ile benzemektedir (Parkhi ve Vedaldi, 2015). Farklı bir çalışmada, yüz tanıma işlemini gerçekleştirmek için araştırmacılar ESA dayalı bir model önermiştirler (Taigman vd., 2014). İlgili çalışmada, siamase mimarisi kullanılmıştır. Önerilen model 4 milyon görüntü ile eğitilip $\% 97.35$ oranında doğruluk performansı sergilemiştir.

Yukarıda ele alınan çalışmalardaki yüz tanıma sistemlerinin performanslarını değerlendirdiğimizde, neredeyse insan seviyesine yakın hatta bazı sistemler daha üstün bir performans sergilediklerini görmekteyiz. Ancak bu denli yüksek performanslı yüz tanıma sistemi oluşturmak için hala büyük zorluklar vardır. Yüksek performanslı sistemlerin eğitildiği veri setlerinin büyüklüğü milyonlarca görüntüden oluşmaktadır. Ancak, açık kaynak olarak paylaşılan veri setlerinin boyutu yüzbinler seviyesindedir. Dolayısıyla bu veri setlerinin boyutunu büyütmek, var olan görüntü sayılarını çoğaltmak için literatür'de değişik veri artırma teknikleri önerilmiştir. Yapılan çalışmada, araştırmacılar, sentetik yüzlerden oluşan yüz görüntülerinin poz, 1şıklandırma ve arkaplanlarını değiştirerek bir veri artırma tekniği önermiştirler (Kortylewski vd., 2018). Yapılan deneyler sonucu poz, aydınlatma ve arka planları değişen görüntülerin yüz tanıma sisteminin performansında olumlu etki ettiği gösterilmiştir. Farklı bir çalışmada, araştırmacılar, yüz görüntülerine farklı gözlük çeşitleri, saç stilleri, aydınlatmalar ve farklı pozlar uygulayarak ilgili veri setinin görüntü sayısını çoğaltmıştırlar (Shao vd., 2017) . Deneyler boyunca bütün veri artırma tekniklerin yüz tanıma sistemindeki performansları ayrı ayrı ele alınmıştır. Deneysel sonuçlar, önerilen tekniklerin yüz tanıma performansını büyük ölçüde iyileştirebileceğini göstermektedir.

$\mathrm{Bu}$ çalışmanın ana amacı, afin dönüşümü (affine transform) veri artırma tekniklerini uygulayarak veri setimizde bulunan görüntülerin sayısını artırarak, ESA dayalı geliştirdiğimiz yüz tanıma sistemindeki etkisini analiz etmektir. Çalışmamızda bütün deneyler Labeled Faces in the Wild (LFW) veri seti üzerinde gerçekleşmiştir.

\section{Materyal ve Yöntem}

Derin öğrenmeye dayalı modeller, son zamanlarda yüz tanıma görevlerinde insan seviyesinin üzerinde bir doğruluk performans1 sergilemektedirler. Hiperparametreler, bu modellerin performans1 üzerinde büyük etkiye sahiptir. Ayrıca, derin modellerin eğitildiği veri setinin boyutu performans üzerinde büyük etkiye sahiptir. $\mathrm{Bu}$ çalışmada yüz tanıma görevi için derin öğrenme tabanlı Evrişimli Sinir Ağı modeli uygulanacaktır. Çalışmamızın ana amacı, afine dönüşümü tekniğini uygulayarak veri setimizdeki görüntü sayısını çoğaltıp yüz tanıma sistemine etkisini analiz etmektir. Çalışmamızın bu bölümde veri seti ve yüz tanıma sistemi hakkında bilgi verilecektir.

\subsection{Veri Seti}


Bu çalışmada kullanılan veri setleri, aşağıda orijinal ve çoğaltılmış veri veri seti olarak tanımlanmıştır. Orijinal veri seti, herhangi bir ön işleme tabi tutulmamış görüntüleri içerir. Çoğaltılmış veri seti ise orijinal veri setindeki görüntüler ve ilgili görüntülere afin dönüşümü uygulanarak elde edilen yeni görüntülerden oluşmaktadır.

\subsection{Orijinal Veri Seti}

Bu çalışmada, Labeled Faces in the Wild (LFW) (Huang vd., 2008) orijinal veri seti olarak kullanılmıştır. İlgili veri seti 5.749 kişiye ait toplamda 13.233 görüntüden oluşmaktadır. İlgili veri setindeki görüntüler web ortamından toplanmıştır. Dolayısıyla, poz, 1şık, yaş vs. gibi özelliklerde bazı farklılıklar vardır. LFW veri setindeki görüntülerin birkaç adeti dışında çoğu görüntü 250 x 250 piksel boyutunda ve renklidir.

\section{3 Çoğaltılmış Veri Seti}

Veri setlerinin boyutu, derin öğrenme ve sınıflandırma modellerini etkilemektedir. Sıfırdan yüz tabanlı veri seti oluşturmak zor ve zaman gerektiren bir problemdir. Çalışmamızın amacı, mevcut görüntülerden afin dönüşümü tekniği ile yeni görüntüler üretip, bu görüntülerin yüz tanıma sistemindeki etkisini analiz etmektir. Afin dönüşümü olarak tanımladığımız teknik, görüntüler üzerinde: yansıma, dönme, ölçekleme ve kırpma işlemlerinin gerçekleşmesidir. Afin dönüşümler, için görüntülerin miktarını artırarak genelde derin sinir ağlarını eğitmek amacıyla kullanılır. Çalışmamızda var olan görüntülere afin dönüşüm uygulamak için Keras kütüphanesinin web sitesinde [Tensorflow Core, 2020] açık kaynak kod'undan yararlandık. Afin dönüşümü uygulandıktan sonra Çoğaltılmış veri setimizde görüntülerin sayısını 19.451 'e çıkarıldı. Şekil 1'de, a.) seçeneği üzerinde Orijinal görüntü gösterilmektedir, b.), c.), d.), e.) ve f.) şıkları üzerinde ise afin dönüşümü ile üretilen yeni görüntüler sirasıyla gösterilmektedir.

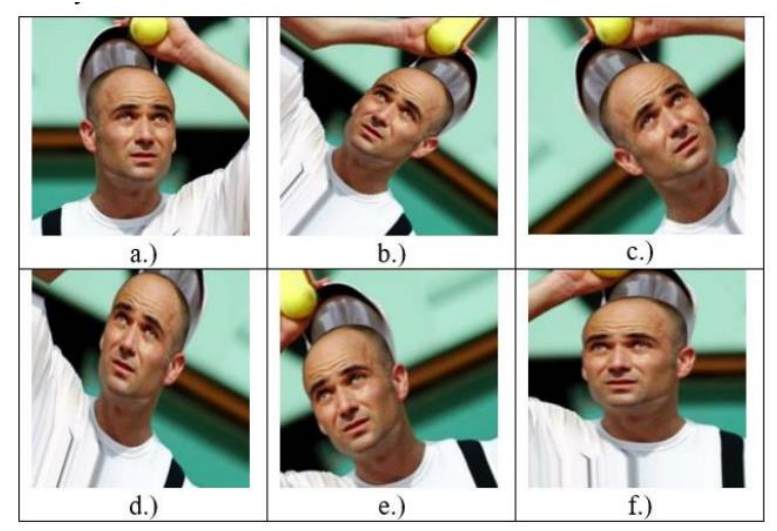

Şekil 1: Afin dönüşümü uygulamadan önce ve sonra örnek görüntüler.

Yüz tanıma görevini gerçekleştirmek için her iki veri setini rastgele olmak üzere eğitim ve test setlerine ayırdık. İlgili veri setlerinin \%80'lik kısımlarını eğitim, kalan \%20'lik bölümü ise test için kullanılmıştır. Her iki veri seti hakkında genel bilgiler Tablo 1.'de gösterilmektedir.
Tablo 1. Çalışmamızda kullanılan veri seti hakkında genel bilgileri göstermektedir.

\begin{tabular}{|l|l|l|}
\hline Veri Seti Türüi & Kişi Sayısı & $\begin{array}{l}\text { Görüntü } \\
\text { Sayısı }\end{array}$ \\
\hline Orijinal Veri Seti & 5.749 & 13.233 \\
\hline Çoğaltılmış Veri Seti & 5.749 & 19.451 \\
\hline
\end{tabular}

\subsection{Yüz Tanıma Sistemi}

Yüz tanıma sistemleri, yüz görüntülerinde veya video karelerinde kişileri tanımlar. Özetle, bir yüz tanıma sistemi, giriş görüntüsünden yüz'e ait özellikleri çıkarır ve bu özellikleri veri setindeki etiketli yüzlerin özellikleriyle karşılaştırır. Çalışmamızda uygulanan yüz tanıma sistemi üç ana aşamadan oluşmaktadır: Yüzün tespit edilmesi, 128 boyutlu vektörün elde edilmesi ve doğrulama/sınıflandırma işlemlerinin gerçekleştirilmesi. Görüntüdeki yüzün tespit edilmesi için Dlib kütüphanesinden faydalanarak Histogram of Oriented Gradient tabanlı yüz tanımlayıcı uygulanarak yüz tespit edilmiştir. HOG algoritması, çeşitli zor koşullar altında yüksek performans sergilemektedir (Déniz vd., 2011). Çalışmamızda girdi görüntülerinin özellikleri çıkarılması için ESA kullanılır. İlgili ESA mimarisi, inception (Szegedy vd., 2015) mimarisinin bir çeşididir. Daha doğrusu, (Schroff vd., 2015)'da NN4 olarak tanımlanan mimari ve OpenFace (Models, 2020) projesinde nn4.small2 olarak tanımlanan modelin bir benzeridir. ESA mimarisinin tamamı (Tim Esler, 2020)'de açıklanmıştır, mimarinin en dikkat çekici tarafı evrişimsel katmanların sonunda 128 gizli düğümden oluşan tam bağlı (fully connected) ve bu katmanın üzerinde bir L2 normalleştirme katmanının (normalization layer) olmasıdır. Tam bağlı katman ve L2 normaleştirme katmanı sayesinde giriş olarak verilen görüntüden yüz'e ait özellikleri barındıran 128 boyutunda vektör elde edilir. Çalışmamızda uygulanan ESA mimarisi oldukça derin bir mimaridir. İlgili mimariyi sıfirdan eğitecek güçlükte bilgisayara sahip olmayışımız, aynı mimariyi önceden eğitilmiş ağırlıklar ile uygulamaya neden olmuştur. Önceden eğitilmiş modelin ağırlıklrı (Models, 2020)'de açık kaynak olarak mevcuttur. Yüz özellikleri vektör halinde elde edildikten sonra doğrulama ve sınıflandırma işlemlerini kolayca gerçekleştirebilmekteyiz. Doğrulama işlemi, literatür'de bire-bir karşılaştırma olarak tanımlanmaktadır. Çalışmamızda, doğrulama işlemi Eşik skor (Treshold score) seçilerek uygulanır, eğer iki görüntü arasında elde dilen değer belirlenen eşik skorun altındaysa pozitif olarak algılanır, dolayısıyla ilgili kişi doğrulanır. Aksi bir durumda negatif olarak algılanır ve kişi yalanlanır. İki görüntü arasındaki değer, yüze ait 128 boyutlu iki vektör arasında Öklid mesafesi [Danielson, 1980] hesaplanarak elde edilir. Gerçekleştirilen deneyler sonucunda mevcut mimari için en uygun eşik değerin 0.64 olduğu görülmüştür. Değerlendirme metriği (Evaluatin metric) olarak F1-skoru kullanılmıştır. Yüz tanıma sisteminin son aşamasında giriş olarak verilen görüntünün veri setindeki hangi kişiye ait olduğunu tespit etmek için sınıflandırma işlemini gerçekleştiriyoruz. ESA mimarisinden 128 boyutlu vektörün elde edilmesi ve en uygun eşik değerin belirlenmesiyle, sınıfandırma işlemi için Makine Öğrenmesine tabanlı Destek Vektör Makinaları (Support Vector Machine) ve K-En Yakın Komşu (KNearest Neighbors) algoritmalarını uyguluyouruz. İlgili algoritmaları yukarıda bahsedilen iki ayrı veri seti ile eğitip, test ettikten sonra elde edilen sonuçları karşılaştırıyoruz. 


\section{Bulgular ve Tartışma}

Çalışmamızda gerçekleşen bütün deneyler, Intel (R) Core(TM) i76700 CPU @3.40 (8 CPUs), Intel (R) HD Graphics 530, 8 RAM donanım gücüne sahip bilgisayarda, Python 3.6 Jupyter Notebook geliştirme ortamı kullanarak geliştirilmiştir. Ayrıca, Keras ve Tensorflow kütüphanelerinden yararlanılmıştır. $\quad \mathrm{Bu}$ çalışmanın ana amacı, afin dönüşüm tabanlı veri büyütme tekniğinin yüz tanıma sistemindeki etkisini analiz etmektir. Doğrulama ve sınıflandırma işlemlerini her iki veri setine ayrı ayrı uygulayarak deneylerimizi gerçekleştiriyoruz. Şekil 2.'de uygulanan sistemin yüz doğrulama işleminde orijinal veri setindeki performansını gösterilmektedir. Şekil 2.'de görüleceği gibi sistem doğrulama işleminde \%92.6 oranında doğruluk elde etti. Şekil 3.'de ise sisteminin doğrulama işleminde çoğaltılmış veri seti üzerinde \%94.4 oranında bir performans sergilediği görülmektedir. Her iki veri seti üzerinde elde edilen sonuçları karşılaştırdığımızda, uygulanan veri artırma tekniği sisteminin doğrulama performansında olumlu yansıdığı ve \%1.8'lik artış sağladığı görülmüştür.

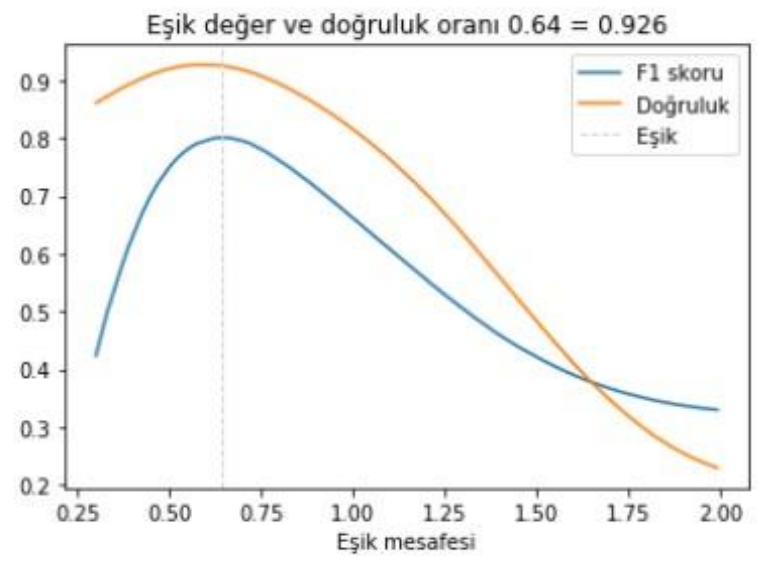

Şekil 2: Sistemin orijinal veri setinde yüz doğrulama performansı.

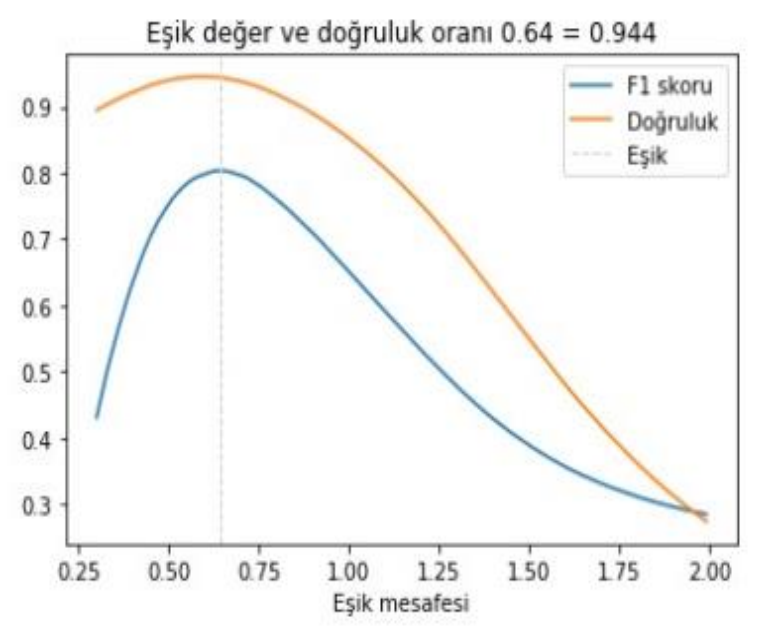

Şekil 3: Sistemin çoğaltılmış veri setinde yüz doğrulama performans1.

Sinıflandırma görevinde Destek Vektör Makineleri (DVM) ve K-En Yakın Komşu (KYK) algoritmalarını iki ayrı veri setine uygulayarak sonuçlar karşılaştırılmıştır. İlgili sonuçlar Tablo 2.'de gösterilmektedir.
Tablo 2. ESA tabanlı yüz tanıma sisteminin sınıflandırma görevinde veri setlerinin karşılaştırılması.

\begin{tabular}{|c|c|c|}
\hline Algoritma & Veri Seti & Doğruluk \% \\
\hline $\begin{array}{c}\text { ESA Tabanlı } \\
\text { Yüz tanıma sistemi } \\
\text { (DVM) }\end{array}$ & Orjinal & 95.5 \\
\cline { 2 - 3 } & Çoğaltılmış & 97.1 \\
\hline $\begin{array}{c}\text { ESA Tabanlı } \\
\text { Yüz tanıma sistemi } \\
(\text { KYK) }\end{array}$ & Orjinal & 95.0 \\
\cline { 2 - 3 } & Çoğaltılmış & 96.2 \\
\hline
\end{tabular}

Sınıflandırma işleminde elde edilen sonuçlara baktığımızda, uygulanan veri artırma tekniği Destek Vektör Makinesi algoritmas1 ile yüz tanıma sisteminin performansında \%1.6 oranında, K-En Yakın Komşu algoritması ise \%1.3 oranında iyileşme sağladığ görülmektedir.

\section{Sonuç}

Çalışmamızda, afin dönüşümü veri artırma tekniğinin yüz tanıma sistemi üzerindeki etkisi analiz edilmiştir. Deneysel sonuçlar, önerilen veri artırma tekniğinin yüz tanıma sistemine olumlu yansıdığı ve doğruluğu artırdığını gerçekleştirilen deneyler ile gösterilmiştir. $\mathrm{Bu}$ artış, yüz doğrulama görevinde $\% 1.8$ oranında olurken, yüz sınıflandırma işleminde Destek Vektör Makineleri algoritması ile \%1.6, K-En Yakın Komşu algoritması ile \%1.3 oranında olmuştur. Sistem, yüz doğrulama işleminde çoğaltılmış veri setinde \%94.4 oranında doğruluk elde ederken, orijinal veri setinde \%92.6 oranında doğruluk elde etti. Sınıflandırma işleminde ise, uygulanan veri artırma tekniği her iki sınıflandırıcıya olumlu yansımıştır. ESA tabanlı sistem Destek Vektör Makineleri sınıflandırıcısı ile orijinal veri setinde $\% 95.5$, çoğaltılmış veri setinde ise \%97.1 oranında performans sergilemiştir. Devamında, ESA tabanlı sistem K-En Yakın Komşu algoritması ile orijinal veri setinde \%.95.0, çoğaltılmış veri setinde ise \%96,3 oranında performans sergilemiştir. Gerçekleştirilen deneyler ve elde edilen sonuçlardan yola çıkarak, çoğaltılmış veri setinin mevcut görüntü sayısını artırdığımız takdirde yüz tanıma sistemin performansında iyileşme olacağının kanaatine varılmıştır.

\section{Kaynaklar}

Fukushima, K. (1988). Neocognitron: A hierarchical neural network capable of visual pattern recognition. Neural networks, 1(2), 119-130.

LeCun, Y., Bottou, L., Bengio, Y., \& Haffner, P. (1998). Gradient-based learning applied to document recognition. Proceedings of the IEEE, 86(11), 2278-2324.

Pawar, K. B., Mirajkar, F., Biradar, V., \& Fatima, R. (2017, September). A novel practice for face classification. In 2017 International Conference on Current Trends in Computer, Electrical, Electronics and Communication (CTCEEC) (pp. 822-825). IEEE.

Kanade, T. (1974). Picture processing system by computer complex and recognition of human faces.

Cox, I. J., Ghosn, J., \& Yianilos, P. N. (1996, June). Feature-based face recognition using mixture-distance. In Proceedings CVPR IEEE Computer Society Conference on Computer Vision and Pattern Recognition (pp. 209-216). IEEE.

Schroff, F., Kalenichenko, D., \& Philbin, J. (2015). Facenet: A unified embedding for face recognition and clustering.

Parkhi, O. M., Vedaldi, A., \& Zisserman, A. (2015). Deep face recognition.

Taigman, Y., Yang, M., Ranzato, M. A., \& Wolf, L. (2014). Deepface: Closing the gap to human-level performance in face verification. In Proceedings of the IEEE conference on computer vision and pattern recognition (pp. 1701-1708). 
Kortylewski, A., Schneider, A., Gerig, T., Egger, B., Morel-Forster, A., \& Vetter, T. (2018). Training deep face recognition systems with synthetic data. arXiv preprint arXiv:1802.05891.

Lv, J. J., Shao, X. H., Huang, J. S., Zhou, X. D., \& Zhou, X. (2017). Data augmentation for face recognition. Neurocomputing, 230, 184-196.

Huang, G. B., Mattar, M., Berg, T., \& Learned-Miller, E. (2008, October). Labeled faces in the wild: A database forstudying face recognition in unconstrained environments. In Workshop on faces in'RealLife'Images: detection, alignment, and recognition.

TensorFlow Core (Kasım, https://www.tensorflow.org/api_docs/python/tf/keras/preprocessing/i mage/ImageDataGenerator.

Déniz, O., Bueno, G., Salido, J., \& De la Torre, F. (2011). Face recognition using histograms of oriented gradients. Pattern recognition letters, 32(12), 1598-1603.

Szegedy, C., Liu, W., Jia, Y., Sermanet, P., Reed, S., Anguelov, D., ... \& Rabinovich, A. (2015). Going deeper with convolutions. In Proceedings of the IEEE conference on computer vision and pattern recognition (pp. 1-9).

Models (Kasım, 2020) https://cmusatyalab.github.io/openface/models-andaccuracies/\#pre-trained-models.

Tim Esler (2020), https://github.com/timesler/facenetpytorch/blob/master/models/inception_resnet_v1.py

Danielsson, P. E. (1980). Euclidean distance mapping. Computer Graphics and image processing, 14(3), 227-248. 